\title{
Seguimiento a 5 años de prótesis híbrida con subestructura sinterizada. Reporte de caso.
}

\section{Five-year follow-up of hybrid prosthesis with sintered substructure. Case report.}

\author{
Natalia Marcus ${ }^{1 *}$, Sebastián González², Michael Wendler ${ }^{1}$, Fernando Grandón", \\ Federico Wernicke ${ }^{1}$, Francisco Marchesani ${ }^{3}$
}

\author{
1. Departamento de Odontología Restauradora, \\ Facultad de Odontología, Universidad de \\ Concepción, Chile. \\ 2. Programa Especialización en Implantología \\ con mención en reconstrucción estética \\ y protésicamente guiada, Universidad de \\ Concepción, Chile. \\ 3. Práctica privada, Concepción, Chile. \\ * Correspondencia Autor: Natalia Marcus | \\ Dirección: Roosevelt 1550, Concepción, Chile| \\ E-mail: nmarcus@udec.cl| Teléfono: +5641266 \\ 1786 - +5641220 4481 \\ Trabajo recibido el 27/05/2020. \\ Trabajo revisado 16/07/2020 \\ Aprobado para su publicación el 25/07/2020
}

\begin{abstract}
RESUMEN
La rehabilitación de rebordes severamente atróficos mediante prótesis híbridas con subestructura cromo-cobalto fabricadas por sinterizado láser asegura el ajuste pasivo, aumentando la estabilidad de los tejidos de soporte a largo plazo. Los controles periódicos y enseñanza de técnicas de higiene son fundamentales para el éxito de rehabilitaciones complejas sobre implantes. El propósito de este reporte de caso es realizar la evaluación clínica y radiográfica a 5 años de prótesis híbridas maxilar y mandibular fabricadas mediante sinterizado láser, encontrándose resultados satisfactorios en la evaluación y seguimiento.
\end{abstract}

PALABRAS CLAVE:

Sinterizado láser; Implantes dentales; Prótesis híbridas.

Int. J. Inter. Dent Vol. 14(3); 285-287, 2021.

\section{ABSTRACT}

Rehabilitation of severely atrophic ridges using hybrid cobalt-chrome substructures manufactured by laser sintering ensures passive fit, increasing support tissue stability. Regular check-ups and the teaching of hygiene techniques are essential for the success of complex implant restorations. The purpose of this case report is to perform a 5-year clinical and radiographic evaluation of hybrid maxillary and mandibular prostheses manufactured using laser sintering, achieving satisfactory results in the evaluation and follow-up.

KEY WORDS:

Laser sintering; Dental implants; Hybrid prostheses.

Int. J. Inter. Dent Vol. 14(3); 285-287, 2021.

\section{INTRODUCCIÓN}

La rehabilitación de rebordes edéntulos mediante tratamientos con implantes dentales ha demostrado ser predecible cuando se presentan volúmenes óseos suficientes ${ }^{(1)}$. Sin embargo, cuando la reabsorción de los maxilares es severa, se presentan limitaciones anatómicas como el patrón de reabsorción centrípeto en el maxilar superior, la neumatización del seno maxilar, la presencia de la fosa nasal y el conducto nasopalatino que dificultan las técnicas quirúrgicas ${ }^{(2)}$. La rehabilitación mediante prótesis híbridas es una alternativa que evita grandes cirugías reconstructivas, logrando dar estabilidad y funcionalidad protésica a pacientes con rebordes atróficos y permitiendo así reemplazar tanto tejidos duros como blandos ${ }^{(3)}$.

Distintos factores van a llevar al éxito a largo plazo de rehabilitaciones protésicas de arcada completa en rebordes severamente atróficas, como son el uso correcto de biomateriales, la habilidad quirúrgica del profesional, la selección de la técnica fabricación de la prótesis híbrida y la mantención profesional y por parte del paciente de rehabilitación protésica ${ }^{(4)}$.

La confección de la subestructura metálica de las prótesis híbridas mediante sinterización láser (SLM) reduce las porosidades, disminuyendo así la susceptibilidad a la corrosión, mostrando además menores niveles de desajuste de la subestructura en comparación con la técnica de colado tradicional(5), lo que se traduce en una menor tensión sobre el tejido periimplantario y en la reducción consecuente del riesgo de reabsorción de hueso crestal ${ }^{(6)}$.

Estudios a largo plazo avalan el uso de implantes inclinados y rehabilitación mediante prótesis híbridas para rehablitación fija de maxilares edéntulos atróficos ${ }^{(7,8)}$. La mayoría de las complicaciones se han asociado más a problemas técnicos cómo fracturas del recubrimiento acrílico o del tornillo protésico que a problemas biológicos, pérdida de los implantes o inflamación de la mucosa periimplantaria( ${ }^{(3)}$.

El éxito a largo plazo de rehabilitaciones complejas de arcadas completas fijas sobre implantes requieren un protocolo de controles periódicos y mantenimiento por parte del paciente. Estos protocolos están pobremente definidos y se basan generalmente en protocolos tradicionales de pacientes dentados o de forma empírica según el tratante ${ }^{(9)}$.

El propósito de este artículo es realizar el reporte de caso a 5 años de una paciente desdentada total rehabilitada mediante prótesis híbridas superior e inferior con subestructura confeccionadas con SLM. Para su evaluación se utilizaron los criterios clínicos de Berglundh ${ }^{(10)}$, que revisa las complicaciones biológicas y técnicas de la rehabilitación sobre implantes.

\section{INFORME DEL CASO}

Paciente de sexo femenino, portadora de prótesis híbrida maxilar y mandibular con data de 5 años desde su inserción. El maxilar superior fue rehabilitado con prótesis híbrida metal-acrílica con subestructura realizada mediante sinterizado láser de Cr-Co con la terminación en acrílico, sobre 7 implantes Zimmer ${ }^{\circledR}$ TSV 3,7 $\times 13$ (Zimmer Dental, Carlsbad, EE. UU.). Los implantes fueron colocados en los sectores laterales en conjunto a elevación de seno bimaxilar para evitar grandes cirugías de reconstrucción en el sector anterior. En el maxilar inferior se realizó la misma rehabilitación 
bajo la técnica quirúrgica Revitalize sobre 4 implantes de la marca anteriormente señalada. Los detalles del caso pueden ser revisado en detalle en Marcus y cols(11).

Desde la inserción de prótesis provisionales fijas, se le indicó a la paciente el uso de irrigador bucal (Waterpik ${ }^{\circledR}$, Dentaid ${ }^{\circledR}$ ) e instrucción de higiene. Una vez dada de alta, fue controlada de manera regular cada 6 meses, recibiendo en cada ocasión higienización mediante el uso de Cavitron $^{\circledR}$, Dentsply Sirona ${ }^{\circledR}$. Ambas prótesis fueron retiradas una vez al año para una limpieza profunda tanto de los pilares como de las prótesis híbridas, mediante ultrasonido, siendo la figura 1 fotografías del control a 5 años previa y posterior a la fase de higienización de ese control (fig. 1.a y 1.b respectivamente). Además del recambio de tornillos protésicos, se examinaron tanto los componentes protésicos como los tejidos periimplantarios, siguiendo la metodología descrita por Berglundh y cols ${ }^{(10)}$. En ninguno de los controles se encontraron fallas en los componentes protésicos (fig. 2), ni en la superestructura o sistema de conexión, no existiendo tampoco algún tipo de alteración sensorial. Los tejidos blandos periimplantarios (fig. 3) tampoco presentaron alteración, no observándose signos de periimplantitis o daño en los implantes ni en los pilares protésicos.

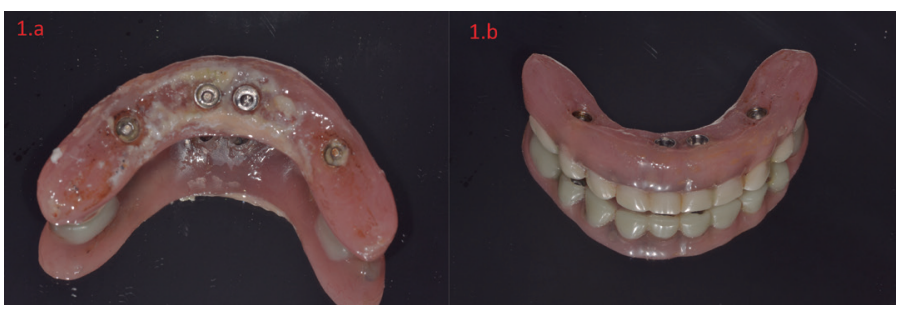

Figura 1. Prótesis híbrida retirada de boca en control 5 años post inserción. 1.a. antes de higiene 1.b. posterior a higienización

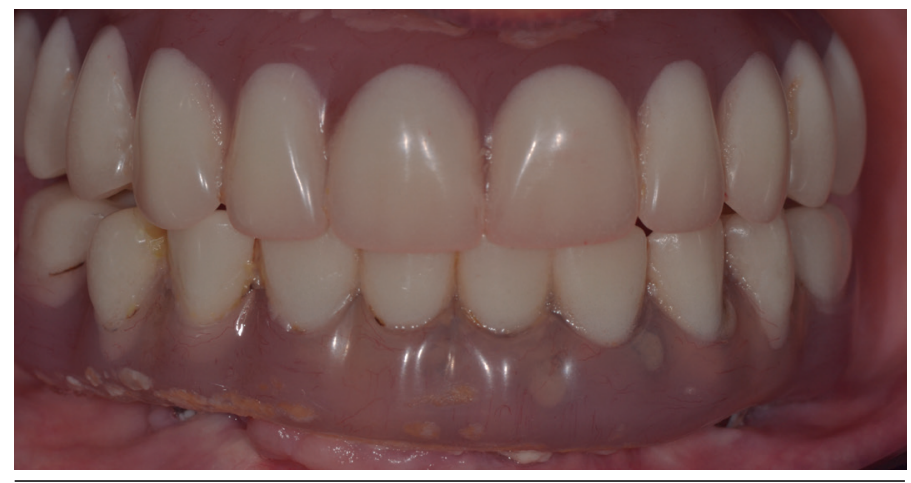

Figura 2. Prótesis híbridas maxilar y mandibular previa higienización en control 5 años

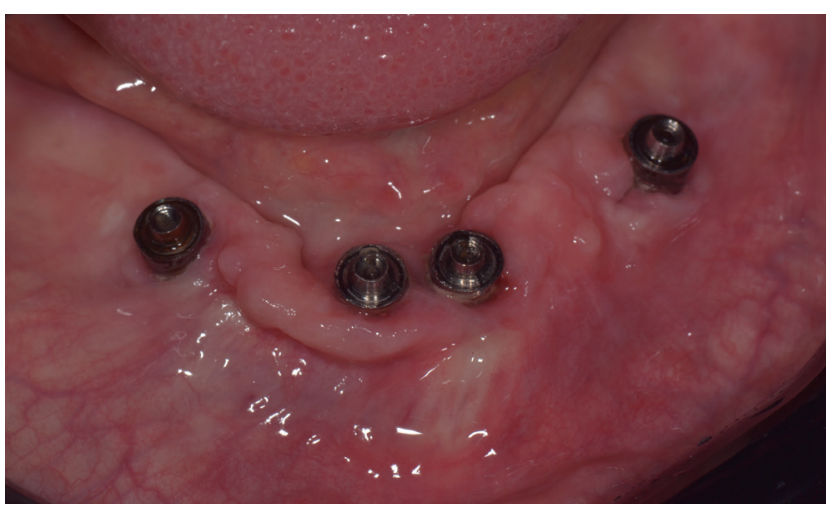

Figura 3. Pilares cónicos sobre implantes mandibulares previa fase de higienización.

La evaluación radiográfica se realizó utilizando tomografías computarizadas (Cone beam CT) y radiografías panorámicas. Para la evaluación del hueso crestal se tomaron como referencia el examen Cone beam CT tomado post rehabilitación y la radiografía panorámica a 5 años (fig. 4). Hay que tener en cuenta que el corte tomográfico utilizado en la figura 4.a sólo muestra una dimensión en su sección coronal y que la radiografía panorámica se obtiene con una superposición de las estructuras presentes y es muy sensible a sufrir distorsión por el posicionamiento del paciente, por lo que pueden existir variaciones en las dimensiones obtenidas. Se observa que en general existe una disminución de la cantidad de hueso en la zona crestal en la comparación de ambas imágenes (fig. 4) tendiendo a ser mayor en la zona maxilar que en la mandibular. Se utilizó el software Romexis para realizar las mediciones.

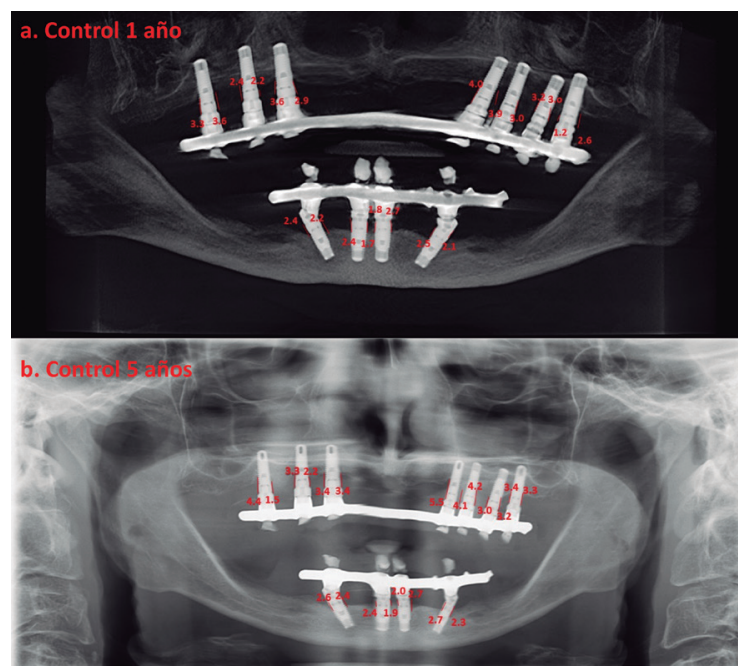

Figura 4. a. corte tomográfico a nivel de los implantes. 4.b. radiografía panorámica control 5 años. Las marcas y números corresponden a la cantidad de hueso crestal alrededor de los implantes en $\mathrm{mm}$. Obsérvese la poca diferencia en la pérdida de hueso crestal desde el primer control a 5 años.

Las figuras 5 y 6 corresponden a los cortes tomográficos obtenidos en el año 2015 en comparación con el año 2019, donde se pude observar la estabilidad dimensional del hueso crestal en los cinco años post alta del paciente y la leve remodelación ocurrida en este período de tiempo.

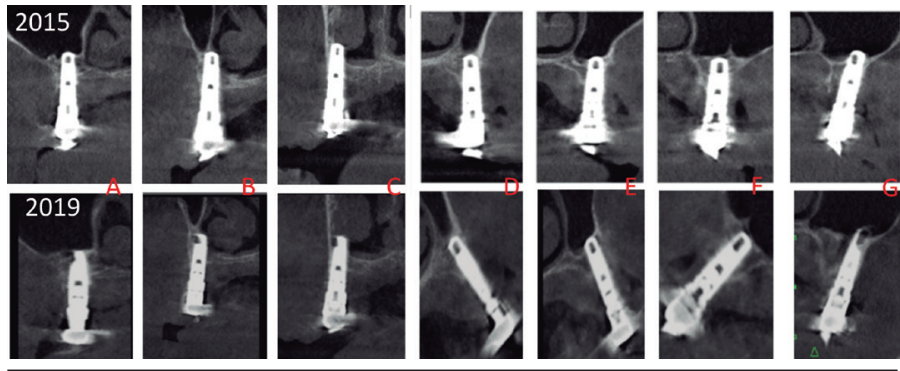

Figura 5. Conebeam maxilar. A. Implante 1.6, B. implante 1.5, C. implante 1.4, D. implante 2.3, E. implante 2.4, F. implante 2.5, G. implante 2.6. se observa la gran estabilidad del hueso en la zona crestal en estos últimos 5 años
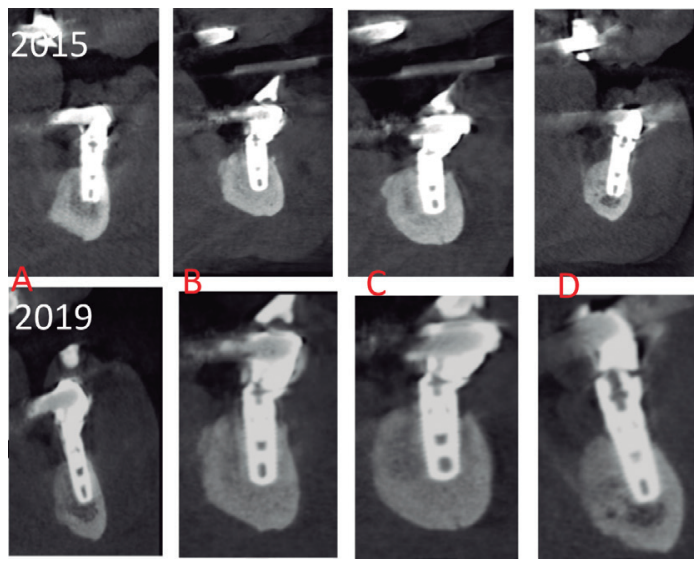

Figura 6. one beam mandibular. A. implante pieza 4.4, B. implante pieza 4.2, C. implante pieza 3.2, D. implante pieza 3.4 . Observe la estabilidad del hueso en la zona crestal 


\section{DISCUSIÓN}

Estudios publicados de implantes rehabilitados mediante estructuras híbridas presentan una tasa de sobrevida alta, entre un $95 \%$ a $100 \%$, independiente de su protocolo de carga ${ }^{(12)}$. En el presente caso clínico, se comprobó que todos los implantes siguen cumpliendo su función, encontrándose una pérdida de hueso a nivel coronal de $3.45 \mathrm{~mm}$ en promedio en el sector maxilar y de $2.4 \mathrm{~mm}$ en promedio en el sector mandibular, desde la cirugía al control de 5 años.

El grado de desadaptación de la subestructura protésica se relaciona de manera directa con la generación de tensiones sobre el tejido periimplantario(13), estando por ello también en directa relación con el éxito de la rehabilitación, lo que sugiere la importancia de conseguir ajuste pasivo de la subestructura, a pesar de que hay estudios que dicen que hay evidencia insuficiente sobre el efecto del desajuste en prótesis atornilladas sobre implantes y los resultados clínicos obtenidos, siendo tema de debate el grado de desajuste tolerable ${ }^{(14)}$. En comparación con el método colado tradicional, la fabricación de una subestructura mediante sinterización láser Cr-Co mejora la adaptación final de la misma y disminuye así las tensiones que se transmiten a los implantes y al hueso que lo rodean ${ }^{(15)}$, lo que facilita un excelente ajuste pasivo, clave en el éxito a largo plazo de la rehabilitación sobre implantes múltiples.

La pérdida de hueso marginal en los implantes fue menor a $2 \mathrm{~mm}$ entre el primer control a los seis meses de alta y el último realizado a cinco años de su inserción, y no se observó una pérdida visible clínicamente de hueso, gracias al cambio de biotipo realizado en la cirugía. Estos resultados concuerdan con el estudio realizado por Friberg y col(16). y se pueden atribuir a la adaptación pasiva de la barra sinterizada y las mantenciones periódicas realizadas.

Los controles periódicos, repaso de técnicas de higiene y retiro de prótesis híbridas con cambio de tornillos protésicos siguieron las recomendaciones dadas por Bidra y $\mathrm{Col}^{(9)}$, que busca mejorar la salud de los tejidos de soporte y limita cualquier proceso patológico aumentando la longevidad de la rehabilitación, abordando tanto las citas de control, el régimen de mantención profesional y en casa de pacientes con restauraciones sobre implantes.

\section{CONCLUSIÓN}

Las prótesis híbridas con una subestructura realizada con sinterizado láser parece tener un buen comportamiento clínico luego de 5 años, especialmente si es acompañada de controles periódicos, instrucción de higiene y seguimiento del paciente de manera regular por parte del equipo tratante para asegurar el éxito y detectar posibles problemas que puedan generar complicaciones en el futuro. Se requiere estudio a mayor plazo para evaluar comportamiento en el tiempo de la rehabilitación sobre implantes.

\section{RELEVANCIA CLÍNICA:}

Existe poca información sobre cómo mantener el éxito a largo plazo de rehabilitaciones híbridas de alta complejidad sobre implantes en rebordes severamente atróficos. Los controles periódicos con fases de higienización, sumado a la fidelización y educación del paciente ayudan a conseguir estabilidad tanto de los tejidos blandos como de la rehabilitación protésica sobre implantes oseointegrados.

\section{Bibliografía}

1. Sorní M, Guarinós J, García O, Peñarrocha M. Implant rehabilitation of the atrophic upper jaw : A review of the literature since 1999. Med Oral Patol Oral Cir Bucal. 2005; 10:45-56.

2. Asharaf S, Suma A. Implant rehabilitation for atrophic maxilla : a review. J Indian Prosthodont Soc. 2014;14(3):196-207.

3. Real-Osuna J, Almendros-Marqués N, Gay-Escoda C. Prevalence of complications after the oral rehabilitation with implant-supported hybrid prostheses. Med Oral Patol Oral Cir Bucal. 2012;17(1):116-21.

4. Drago C, Gurney L. Maintenance of Implant hybrid prostheses : clinical and laboratory procedures. J Prosthodont. 2013;22(1):28-35.

5. Koutsoukis T, Zinelis S, Eliades G, Al-Wazzan K, Rifaiy M, Al Jabbari Y. Selective laser melting technique of co-cr dental alloys: a review of structure and properties and comparative analysis with other available techniques. J Prosthodont. 2015;24(4):303-12

6. de Araújo G, de França D, Silva Neto J, Barbosa G. Passivity of conventional and CAD/CAM fabricated implant frameworks. Braz Dent J. 2015;26(3):277-83.

7. Maló P, de Araújo Nobre M, Lopes A, Moss SM MG. A longitudinal study of the survival of All-on-4 implants in the mandible with up to 10 years of follow-up. J Am Dent Assoc. 2011:142:310-20.

8. Kwon T, Bain PA, Levin L. Systematic review of short- (5-10 years) and long-term (10 years or more) survival and success of full-arch fixed dental hybrid prostheses and supporting implants. J Dent. 2014;42(10):1228-41.

9. Bidra AS, Daubert DM, Garcia LT, Nenn CA, Olsen JA, Platt JA, et al Clinical practice guidelines for recall and maintenance of patients with toothborne and implant-borne dental restorations. J Prosthodont. 2016;25(1):32-40.
10. Berglundh T, Persson L. A systematic review of the incidence of biological and technical complications in implant dentistry reported in prospective longitudinal studies of at least 5 years. J Clin Periodontol. 2002;29(3):197-212.

11. Marcus Vaccarezza N, Marchesani Carrasco F, Grandón Villegas F, Galdames Gutiérrez B, Niklitschek Tapia D, Millanao Caro L. Rehabilitación de rebordes severamente atróficos mediante prótesis híbridas confeccionadas con tecnología de sinterización láser cromo-cobalto; reporte de caso. Rev Clínica Periodoncia Implantol y Rehabil Oral. 2016;9(1):13-8.

12. Gallardo Y, da Silva-Olivio I, Gonzaga L, Sesma N, Martin W. A systematic review of clinical outcomes on patients rehabilitated with complete-arch fixed implant-supported prostheses according to the time of loading. J Prosthodont. 2019;28(9):958-68

13. Taşın S, Turp I, Bozdağ E, Sünbüloğlu E, Üşümez A. Evaluation of strain distribution on an edentulous mandible generated by cobalt-chromium metal alloy fixed complete dentures fabricated with different techniques: An in vitro study. J Prosthet Dent. 2019;122(1):47-53

14. Katsoulis J, Takeichi T, Gavira A, Peter L, Katsoulis K. Misfit of implant prosthesis and its impact on clinical outcomes. Definition, assessment and a systematic review of the literature. Eur J Oral Implantol 2017; 10(Suppl 1):121-38.

15. Fernández M, Delgado L, Molmeneu M, García D, Rodríguez D. Analysis of the misfit of dental implant-supported prostheses made with three manufacturing processes. J Prosthet Dent. 2014;111(2):116-23.

16. Friberg B, Jemt $T$. Rehabilitation of edentulous mandibles by means of osseointegrated implants: a 5-year follow-up study on one or two-stage surgery, number of implants, implant surfaces, and age at surgery. Clin Implant Dent Relat Res. 2015;17(3):413-24. 